\title{
Exceptional points for photon pairs bound by nonlinear dissipation in cavity arrays
}

\author{
Mark Lyubarov ${ }^{1, *}$ and Alexander Poddubny $2,1,3$ \\ ${ }^{1}$ Physics and Engineering Department, ITMO University, St. Petersburg 197101, Russia \\ ${ }^{2}$ Nonlinear Physics Centre, Australian National University, Canberra ACT 2601, Australia \\ ${ }^{3}$ loffe Institute, St. Petersburg 194021, Russia \\ *markljubarov@gmail.com
}

Compiled September 13, 2018

We study theoretically the dissipative Bose-Hubbard model describing array of tunneling-coupled cavities with non-conservative photon-photon interaction. Our calculation of the complex energy spectrum for the photon pairs reveals exceptional points where the two-photon states bound by nonlinear dissipation are formed. This improves fundamental understanding of the interplay of non-Hermiticity and interactions in the quantum structures and can be potentially used for ondemand nonlinear light generation in photonic lattices. (C) 2018 Optical Society of America

http://dx.doi.org/10.1364/ao.XX.XXXXXX

Nonlinear integrated optical circuits are a promising platform for quantum information processing and already reveal fundamental quantum effects [1-5]. One of the simplest interaction effects in a quantum system is a formation of two-boson pairs as a result of repulsive interaction, originating from the Kerr-like nonlinearity [6], demonstrated for the first time in a cold atom system [7]. Quantum two-photon effects are also possible in the superconducting resonator networks [8]. Another potential realization is an array of coupled nonlinear cavities, where the two-photon edge states mediated by interaction have been predicted [9-15]. The system of two interacting photons in a one-dimensional array of nonlinear cavities can be also emulated classically using a two-dimensional array of linear cavities [16-18]. While the role of dissipation and dephasing in this system has been studied [19], to the best of our knowledge, the formation of bound two-particle states has been considered only due to a purely Hermitian, conservative, interaction. Spatially modulated losses (or gain) can lead to a dramatic modification of the structure properties and significantly expand the domain of Hermitian systems [20]. In particular, the parity-time symmetric structures [21], where losses and gain are symmetrically distributed in space, are beneficial for single mode lasing [22-25]. $\mathcal{P} \mathcal{T}$-symmetry and non-Hermiticity can be also used to design topological edge states of light, protected from the disorder [2628 ] and promising for guiding of waves on a chip.

Here, we examine the effect of dissipative interactions on the behavior of photon pairs in the array of nonlinear cavities. Formally, we consider a Bose-Hubbard model with the imagi-

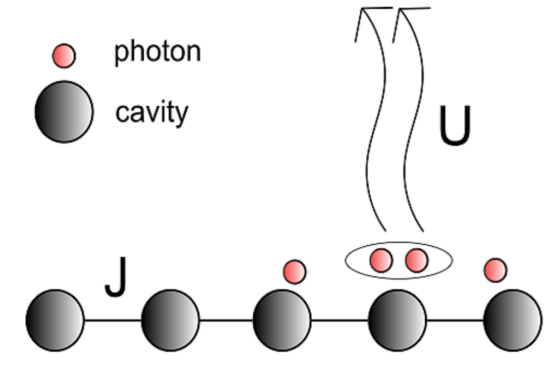

Fig. 1. Scheme of the array of coupled cavities with the tunneling constant $J$ and dissipative photon-photon interaction $U$.

nary interaction term. This can be realized in practice, e.g., by introducing the dissipative Kerr nonlinearity, leading to the absorption of photon pairs located in the same cavity. The structure under consideration is schematically illustrated in Fig. 1. It is described by the Hamiltonian

$$
\begin{array}{r}
H=H_{0}+U \equiv \hbar \omega_{0} \sum_{j=1}^{N} a_{j}^{\dagger} a_{j}+J \sum_{j=1}^{N-1}\left(a_{j}^{\dagger} a_{j+1}+a_{j+1}^{\dagger} a_{j}\right) \\
-\mathrm{i} U \sum_{j=1}^{N} a_{j}^{\dagger} a_{j}\left(a_{j}^{\dagger} a_{j}-1\right),
\end{array}
$$

where $a_{j}^{\dagger}\left(a_{j}\right)$ are the photon creation (annihilation) operators, $J$ is the tunneling parameter and $U$ is the dissipative interaction strength.

We look for the two-photon solutions of the Hamiltonian (1) where the wavefunction has the form

$$
|\Psi\rangle=\sum_{j, j^{\prime}=1}^{N} \Psi_{j j^{\prime}} a_{j}^{\dagger} a_{j^{\prime}}^{\dagger}|0\rangle,
$$

with $\Psi_{j j^{\prime}}=\Psi_{j^{\prime} j}$ reflecting the bosonic nature of the excitations. We substitute the wavefunction (7) in the Schrödinger equation $E \Psi=H \Psi$ with the Hamiltonian (1) and obtain a system of linear equations for the coefficients $\Psi_{j j^{\prime}}$ in Eq. (7). As such, the interacting two-particle problem in one dimension is exactly mapped to the noninteracting single-particle problem in two 
dimensions [17]. Solution of the system of equations yields the complex energy spectrum of the finite structure. We stress, that the proposed description of the non-Hermitian system by a Schrödinger equation with a damping term is approximate. More rigorous model would require the formalism of master equation for the density matrix or an explicit account for the reservoir describing the losses [29]. However, our simplistic approximation already allows us to capture the competition between the nonlinear dissipative losses and tunneling-induced coupling.

Two types of photon pair eigenstates can be expected. The first type describes the so-called scattering states, i.e. pairs where two photons are delocalized and quasi-independent from each other. Their energy is given by a sum of two independent terms corresponding to non-interacting single-photon states. The second type corresponds to bound photon pairs (doublons), where two photons are localized near each other. For the infinite structure the dispersion of these pairs is described by the following equation

$$
E(k)=-2 \sqrt{4 J^{2} \cos ^{2} k-U^{2}},
$$

where $k$ is the center-of-mass wave vector, $\Psi_{j j^{\prime}} \propto \exp \left[i k\left(j+j^{\prime}\right) / 2\right]$. Equation (3) was obtained by an analytical continuation of the result in [7] to the case of complex photon-photon interaction strength. It indicates that the energy spectrum should exhibit a transition for $U=2 J$, and has exceptional points at $k= \pm \arccos U /(2 J)$ for $|U|<2|J|$ when the expression under the square root vanishes. We will now verify this by a rigorous calculation for a finite structure. Figure 2(a) shows the energy spectrum calculated as a function of the interaction strength. It can be seen, that the system exhibits a transition for $U=2 J$. For $U<2 J$ there is one energy band while for $U>2 J$ the spectrum splits into two separate bands. The upper one corresponds to doublons, bound by a dissipative interaction. Its energies lie between two asymptotic values

$$
E=-2 i U
$$

and

$$
E=-2 \mathrm{i} \sqrt{U^{2}-4 J^{2}},
$$

corresponding to the dispersion of the infinite structure Eq. (3) with $k=\pi / 2$ (blue curve in Fig. 2(a)) and $k=0$ (orange curve), respectively.

The lower band corresponds to quasi-independent photons, i.e. scattering states. It is instructive to estimate the damping of these states as a function of the dissipation $U$ and a number of cavities $N$. There exist $\sim N^{2}$ such states, each state is distributed over the whole $N \times N$ square, so $\Psi_{j j^{\prime}} \propto 1 / N$. At the limit of $U \gg$ $2 J$ we have $|E| \ll U$, and the diagonal cells $\left(j=j^{\prime}\right)$ are classically forbidden. The imaginary part of energy is responsible for the losses and can be estimated by the perturbation theory. The losses are proportional to the part of the wave function located on the diagonal and are due to the mixing of quasi-independent and doublon bands. The matrix element, responsible for the mixing, is on the order of $J$ and the (imaginary) energy mismatch is $\sim U$. Thus the damping of quasi-independent photons is $\operatorname{Im}(E) \sim J^{2} / N U$. More precise asymptotic is provided by an heuristic expression,

$$
\operatorname{Im} E=-\frac{C J^{2}}{N \sqrt{U^{2}-4 J^{2}}},
$$

where $C \approx 7.66$, that accounts for the transition point at $U=$ $2 J$ and well agrees with the numerical calculation in Fig. 2(a)
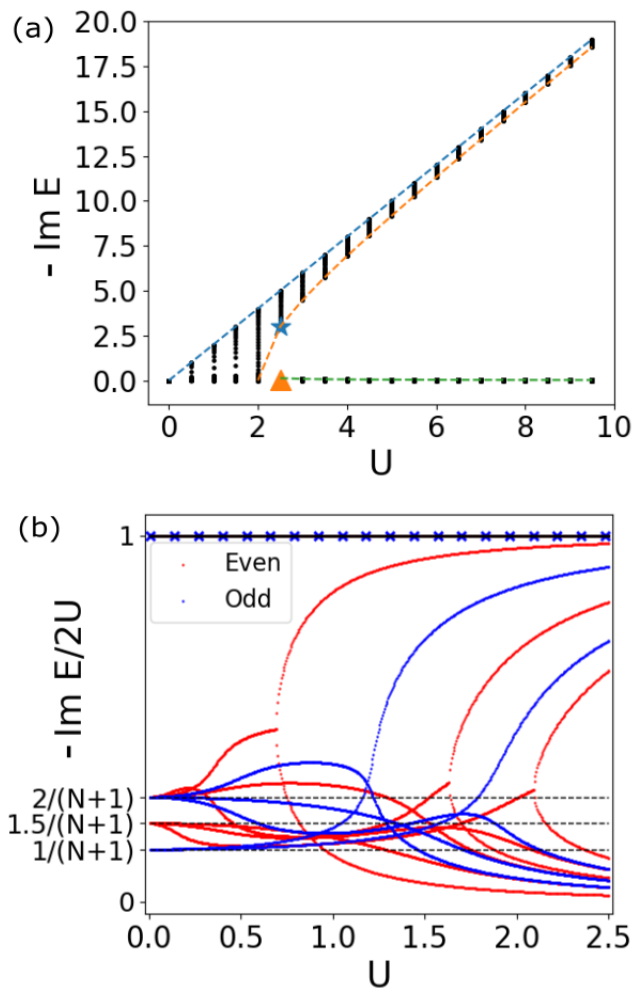

Fig. 2. Dependence of the imaginary component of the spectrum on the dissipative interaction strength. (a) Solid circles show the numerical calculation for a finite array; blue, orange and green lines present asymptotic results Eq. (4), Eq. (5),Eq. (6), respectively. Calculation has been performed for $N=30$ cavities and $J=1$. (b) Same as (a) but in a larger scale for $N=6$ cavities and normalized to the interaction strength. Red and blue circles correspond to the modes of different parity.

(green curve). Interestingly, Eq. (6) indicates that the losses vanish, $\operatorname{Im} E \rightarrow 0$, when we consider an infinite system, $N \rightarrow$ $\infty$. This is because the relative contribution of the doublon states, occupying only the diagonal of the $N \times N$ Hilbert space, becomes weak.

As expected, the decrease of the interaction strength leads to delocalization and destruction of the doublon modes. However, the transition between the regimes with separate doublon and quasi-independent bands $(U \gg 2 J)$ and mixed bands $(U \ll 2 J)$ is rather intricate. For small $N$ the transition takes place at different values of $U$ for different doublon modes. Figure 2(b) shows the energy spectrum in the region $0<U<2.5 \mathrm{~J}$. We consider a small structure with $N=6$ cavities, so that the behavior of all the modes can be traced. The calculation demonstrates that the fate of the doublons at small $U$ depends on their parity with respect to the transformation $j \rightarrow N+1-j, j^{\prime} \rightarrow N+1-j^{\prime}$. The odd modes (blue circles) smoothly change their energy with the interaction strength and delocalize. The even modes (red circles) exhibit exceptional points at different values of $U$. When the interaction strength becomes weaker than the position of the exceptional point, they coalesce with the scattering states and delocalize.

More insight into the behavior at $U \ll J$ can be obtained by considering the dissipative interaction as a perturbation. The eigenstates at $U=0$, describing a pair of non-interacting pho- 
tons, read

$$
\left|\Psi\left(k_{1}, k_{2}\right)\right\rangle=\sum_{j, j^{\prime}=1}^{N} C_{k_{1}, k_{2}}\left(\sin k_{1} j \sin k_{2} j^{\prime}+\sin k_{1} j^{\prime} \sin k_{2} j\right)\left|j, j^{\prime}\right\rangle,
$$

where $k_{i}=\pi l_{i} /(N+1), l_{1}=1, \ldots, N, l_{2}=1, \ldots, l_{1}$, and $\left|j, j^{\prime}\right\rangle$ is the state with photons in cavities $j$ and $j^{\prime}$. The normalization coefficients are $C_{k_{1}, k_{2}}=\sqrt{1+\delta_{k_{1}, k_{2}}} /(N+1)$. There exist $N(N+$ $1) / 2$ such states, since $\left|\Psi\left(k_{1}, k_{2}\right)\right\rangle \equiv\left|\Psi\left(k_{2}, k_{1}\right)\right\rangle$. They can be distinguished based on their symmetry and the degeneracy of their spectrum $E\left(k_{1}, k_{2}\right)=2 J\left(\cos k_{1}+\cos k_{2}\right)$.into three groups with $k_{1}=k_{2}$, with $k_{1}+k_{2}=\pi$ and with $k_{1} \neq k_{2}$.

The $N$ states with $k_{1}=k_{2}$ are non-degenerate at $U=0$. The linear-in- $U$ energy correction is

$$
E=\mathrm{i}\left\langle\Psi\left(k_{1}, k_{2}\right)|U| \Psi\left(k_{1}, k_{2}\right)\right\rangle=-i \frac{3 U}{2(N+1)} .
$$

For the states with $k_{1} \neq k_{2}$ the spectrum is non-degenerate as well and the first order energy correction is equal to

$$
E=-2 \mathrm{i} U /(N+1) .
$$

There exist $N(N-1) / 2$ and $(N-1)^{2} / 2-1$ such states for even (odd) $N$, respectively.

The most interesting case is presented by the remaining states with $k_{1}=k_{2}=\pi$. They all have energy $E=0$ and the degeneracy $N / 2(N / 2+1 / 2)$ for even (odd) $N$. It is convenient to rewrite Eq. (7) for these states as

$$
|\Psi(k)\rangle=\frac{\sqrt{2}}{N+1} \sum_{j, j^{\prime}=1}^{N} \sin k j \sin k j^{\prime}\left[(-1)^{j+1}+(-1)^{j^{\prime}+1}\right]\left|j, j^{\prime}\right\rangle
$$

where $k_{1} \equiv k$ and $k_{2} \equiv \pi-k$. In such notation it becomes clear that for even $N$ all these states are odd. Using this wavefunction we obtain the matrix elements of the perturbation as

$$
V_{k k^{\prime}} \equiv \mathrm{i}\left\langle\Psi(k)|U| \Psi\left(k^{\prime}\right)\right\rangle=\frac{\mathrm{i} U}{N+1}\left(2+\delta_{k k^{\prime}}\right) .
$$

Solving the secular equation $\left|V_{k k^{\prime}}-E \delta_{k k^{\prime}}\right|=0$ we finally obtain $N / 2$ solutions, including

$$
\begin{aligned}
& E=-\mathrm{i} U /(N+1) \\
& E=-\mathrm{i} U \\
& (N / 2-1) \text { states } \\
& 1 \text { state . }
\end{aligned}
$$

All these modes evolve into doublons with the increase of the interaction strength. The analytical results Eq. (8),Eq. (9),Eq. (12),Eq. (13) are shown as horizontal lines in Fig. 2(b) and agree with the numerical calculation for $U \ll J$.

Figure 3 shows the wave functions in the real space. Two coordinates in these maps are responsible for the cavity numbers of the two photons, $j$ and $j^{\prime}$. We consider case with strong interaction, namely $U=2.5 \mathrm{~J}$, so that states from both the doublon band and the band with quasi-independent photons can be illustrated. In Fig. 3(b,c) the two states from the doublon band are presented. Wave function decays away from the diagonal $j=j^{\prime}$, which means that two photons are localized near each other, as expected from a doublon state. In Fig. 3(a) the wave function is distributed over all $N \times N$ square and no localization is observed.

As discussed above, the energy dispersion law (3) of the infinite periodic structure has exceptional points for $|U|<2|J|$ for certain values of wave vectors. For finite $N$ the translational
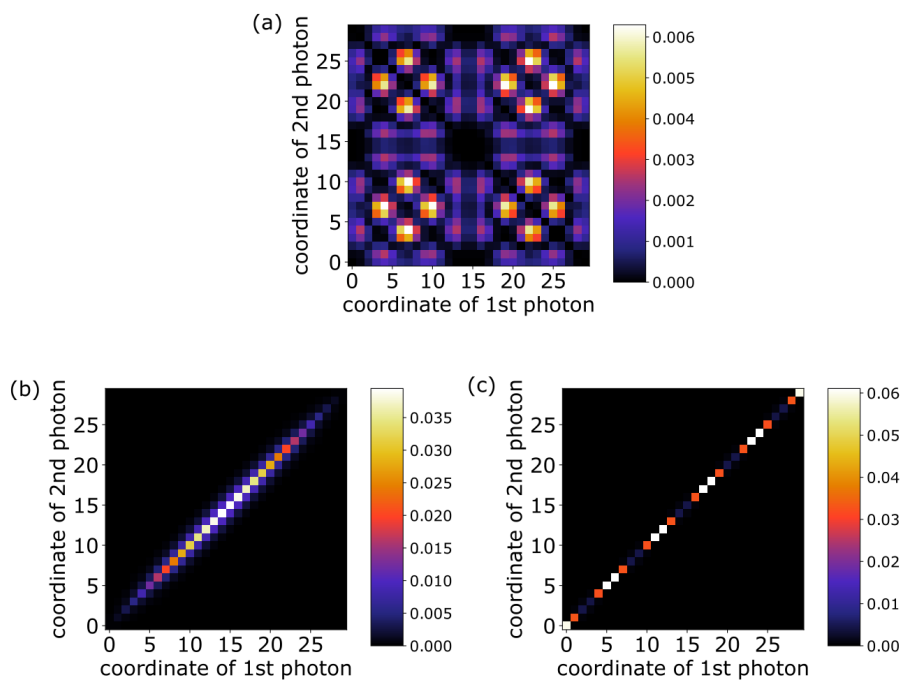

Fig. 3. Illustration of the two-photon wave function for bulk (a) and bound (b), (c) states with $U=2.5 \mathrm{~J}$, and the energies $E \approx(3.01-0.04 \mathrm{i}) J, E \approx-3.007 \mathrm{i} J$ and $E \approx-4.89 \mathrm{i} J$, respectively. Other calculation parameters are the same as in Fig. 2(a).

invariance is broken and the the wave-vector. It is then an instructive question whether these exceptional points in reciprocal space can be directly traced for a finite cavity array. To this end we perform the Fourier transformation of the eigenmodes $\Psi^{(v)}$ of the finite structure [30]:

$$
\widetilde{\Psi}_{s}^{(v)}(k)=\sum_{l=s}^{n} \Psi_{l, l-s}^{(v)} e^{-\mathrm{i} k\left(l-\frac{s}{2}\right)}
$$

to obtain the amplitudes $\Psi_{s}^{(v)}(k)$ depending on the photonphoton distance $s$ and the center-of-mass wave vector $k$ and the mode number $v$. Next, we introduce the wave-vector-resolved density of states $F(E, k)$ for the distribution of photon pairs, located in the same cavity,

$$
F(E, k)=\sum_{v} e^{-\frac{\left|E-E_{v}\right|^{2}}{\sigma^{2}}}\left|\widetilde{\Psi}_{0}^{(v)}(k)\right|^{2}
$$

Figure 4 presents the calculated complex energy spectrum (left column) along with the wave-vector-resolved density of states in the doublon bands (right column) for three values of the dissipative interaction strength, $U=1.5 \mathrm{~J}, U=2 \mathrm{~J}$, and $U=2.5 \mathrm{~J}$. The density of states, extracted from Eq. (15), agrees well with the analytical dispersion Eq. (3) for the infinite structure, which means that for $N=31$ cavities the doublon wave vector is already a good quantum number. In agreement with the calculation in Fig. 3 the spectrum demonstrates a qualitative transition at at $U=2 J$. While for strong interaction the doublons are defined for all values of wavevector and their dispersion resembles a typical cosine function [Fig. 4(b)], for weak interaction, $U<2 J$, the doublons are defined only for larger wavevectors $|k|>\arccos U /(2 J)$ and their dispersion features an exceptional point [Fig. 4(f)]. The same transition can be traced for the complex energy spectrum: for strong interaction the doublon band is spectrally separated in the complex plane from the band of quasi-independent photons [Fig. 4(e)] and for weak interaction the doublons merge with quasi-independent photons with the spectral distance decreasing at large $N$ [Fig. 4(a)]. 
(a)

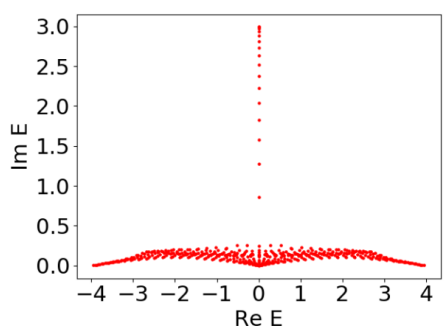

(c)

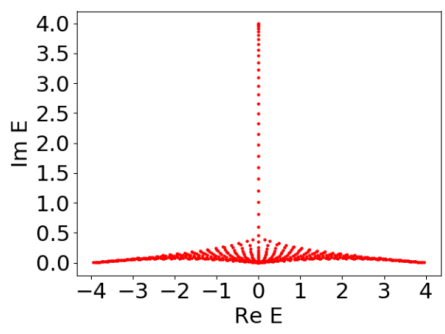

(e)

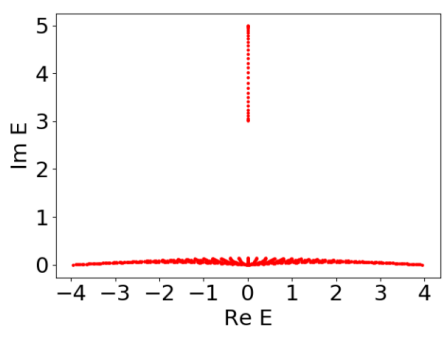

(b)

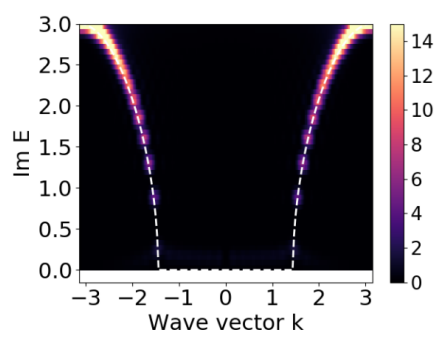

(d)

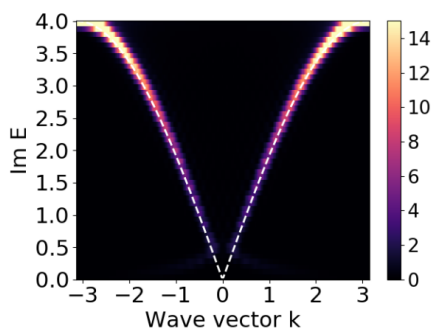

(f)

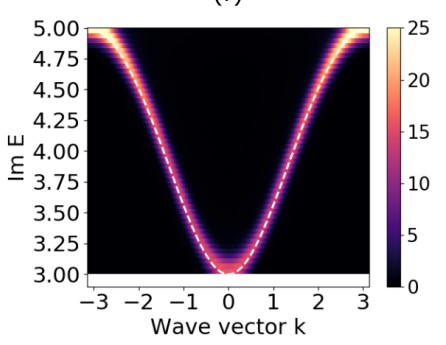

Fig. 4. (a,c,e) Complex energy spectrum for $U=1.5 \mathrm{~J}$ (a), $U=2 J(\mathrm{c}), U=2.5 J(\mathrm{e})$. Panels $(\mathrm{b}, \mathrm{d}, \mathrm{f})$ show density of states in the reciprocal space for corresponding values of $U$ calculated following Eq. (14). Solid white lines show the analytical dispersion calculated from Eq. (3). Other calculation parameters are the same as in Fig. 2.

To summarize, we have considered two-photon states in the array of nonlinear cavities with dissipative Kerr-like nonlinearity within the framework of non-Hermitian Bose-Hubbard model. We demonstrate, that the strong dissipative nonlinearity can lead to the formation of well-defined spatially-bound two-photon states. Such doublons have finite lifetime and correspond to photon pairs localized in the same cavity. In case of weak nonlinearity, the formation of the doublon states is also possible for large center-of-mass wave vectors close to the edge of the Brillouin zone. For lower wave-vectors the doublon energy spectrum features interaction-driven exception points, where the doublons merge with the quasi-independent two-photon scattering states.

In experiment, if the dissipative interaction is induced by the sum-frequency generation or two-photon absorption processes, the formation of the doublon state would be manifested by shortliving traces of the sum-frequency emission from the same cavity. However, for strong dissipative interaction the lifetime of the doublons becomes too short, while for weak interaction they become delocalized. Hence, we expect that there exists an optimal value of the dissipation when the experimental signature of the doublons would be the strongest. We hope that our results will be useful for emerging studies of quantum, active and topological photonic structures $[4,5,31]$.

We acknowledge useful discussions with M.A. Gorlach, A. Blanco-Redondo and M. Di Liberto. This work was supported by the Russian Science Foundation (Grant No. 16-19-10538). A.N.P. acknowledges support from the Australian Research Council.

\section{REFERENCES}

1. I. Carusotto and C. Ciuti, Rev. Mod. Phys. 85, 299 (2013).

2. A. Peruzzo, M. Lobino, J. C. F. Matthews, N. Matsuda, A. Politi, K. Poulios, X.-Q. Zhou, Y. Lahini, N. Ismail, K. Worhoff, Y. Bromberg, Y. Silberberg, M. G. Thompson, and J. L. OBrien, Science. 329, 1500 (2010).

3. A. S. Solntsev, F. Setzpfandt, A. S. Clark, C. W. Wu, M. J. Collins, C. Xiong, A. Schreiber, F. Katzschmann, F. Eilenberger, R. Schiek, W. Sohler, A. Mitchell, C. Silberhorn, B. J. Eggleton, T. Pertsch, A. A. Sukhorukov, D. N. Neshev, and Y. S. Kivshar, Phys. Rev. X 4, 031007 (2014).

4. S. Mittal and M. Hafezi, ArXiv e-prints, 1709.09984 (2017).

5. S. Barik, A. Karasahin, C. Flower, T. Cai, H. Miyake, W. DeGottardi, M. Hafezi, and E. Waks, Science 359, 666 (2018).

6. D. C. Mattis, Rev. Mod. Phys. 58, 361 (1986).

7. K. Winkler, G. Thalhammer, F. Lang, R. Grimm, J. H. Denschlag, A. J. Daley, A. Kantian, H. P. Büchler, and P. Zoller, Nature. 441, 853 (2006).

8. P. Roushan, C. Neill, A. Megrant, Y. Chen, R. Babbush, R. Barends, B. Campbell, Z. Chen, B. Chiaro, A. Dunsworth, A. Fowler, E. Jeffrey, J. Kelly, E. Lucero, J. Mutus, P. J. J. O'Malley, M. Neeley, C. Quintana, D. Sank, A. Vainsencher, J. Wenner, T. White, E. Kapit, H. Neven, and J. Martinis, Nat. Phys. 13, 146 (2016).

9. R. Pinto, M. Haque, and S. Flach, Phys. Rev. A 79, 052118 (2009).

10. S. Longhi and G. D. Valle, J. Physics: Condens. Matter 25, 235601 (2013).

11. M. Di Liberto, A. Recati, I. Carusotto, and C. Menotti, Phys. Rev. A 94, 062704 (2016).

12. M. A. Gorlach and A. N. Poddubny, Phys. Rev. A 95, 053866 (2017).

13. M. Di Liberto, A. Recati, I. Carusotto, and C. Menotti, Eur. Phys. J. Special Top. 226, 2751 (2017).

14. M. A. Gorlach and A. N. Poddubny, Phys. Rev. A 95, 033831 (2017).

15. G. Salerno, M. Di Liberto, C. Menotti, and I. Carusotto, Phys. Rev. A 97, 013637 (2018).

16. A. Schreiber, A. Gábris, P. P. Rohde, K. Laiho, M. Štefaňák, V. Potoček, C. Hamilton, I. Jex, and C. Silberhorn, Science 336, 55 (2012).

17. G. Corrielli, A. Crespi, G. Della Valle, S. Longhi, and R. Osellame, Nat. Commun. 4, 1555 (2013).

18. M. A. Gorlach, M. Di Liberto, A. Recati, I. Carusotto, A. N. Poddubny, and C. Menotti, ArXiv e-prints, 1808.05989 (2018).

19. M. Bello, G. Platero, and S. Kohler, Phys. Rev. B 96, 045408 (2017).

20. G. Borrmann, Zeitschrift für Physik 127, 297 (1950).

21. L. Feng, R. El-Ganainy, and L. Ge, Nat. Photonics 11, 752 (2017).

22. L. Feng, Z. J. Wong, R.-M. Ma, Y. Wang, and X. Zhang, Science 346, 972 (2014).

23. H. Hodaei, M.-A. Miri, M. Heinrich, D. N. Christodoulides, and M. Khajavikhan, Science 346, 975 (2014).

24. A. V. Poshakinskiy, A. N. Poddubny, and A. Fainstein, Phys. Rev. Lett. 117, 224302 (2016).

25. L. Pilozzi and C. Conti, Opt. Lett. 42, 5174 (2017).

26. S. Weimann, M. Kremer, Y. Plotnik, Y. Lumer, S. Nolte, K. G. Makris, M. Segev, M. C. Rechtsman, and A. Szameit, Nat. Mater. 16, 433 (2017).

27. D. Leykam, K. Y. Bliokh, C. Huang, Y. D. Chong, and F. Nori, Phys. Rev. Lett. 118, 040401 (2017).

28. X. Ni, D. Smirnova, A. Poddubny, D. Leykam, Y. Chong, and A. B. Khanikaev, ArXiv e-prints, 1801.04689 (2018).

29. A. Kavokin, J. Baumberg, G. Malpuech, and F. Laussy, Microcavities (Clarendon Press, Oxford, 2006).

30. P. Hapala, K. Kůsová, I. Pelant, and P. Jelínek, Phys. Rev. B 87, 195420 (2013).

31. A. N. Poddubny and D. A. Smirnova, ArXiv e-prints, 1808.04811 (2018). 